\title{
Brachytherapy in reirradiation of locally recurrent nasopharyngeal cancer: case report
}

\begin{abstract}
Purpose: Nasopharyngeal carcinoma (NPC) is a most cancer of head and neck in North Africa and South East Asia. The risk of local recurrence after treatment is higher. Reirradiation can be difficult and limited by the constraints of doses to organs at risk already irradiated. The aim of this study was to determine the role of brachytherapy in safety reirradiation of recurrent NPC.
\end{abstract}

Methods and Materials: From 2002 to 2011, five patients were reirradiated for local recurrence nasopharyx cancer with or without external beam radiotherapy (EBRT).

Results: The median age was 49years, all were male, four patients had World Health Organization Class III histology, and one with nasopharyx cancer type I. Two, 1 and 2 patients had recurrent Stage I, II, III, respectively. Median time to reirradiation was 49 months. Four patients received combined-modality treatment, consisting of external beam radiotherapy (EBRT) with chemotherapy followed by intracavitary brachytherapy High Dose Rate (HDR) boost, one patient received intracavitary brachytherapy alone for dosimetric constraints for organs at risk. The total radiation dose EQD2 was between 60 and $70 \mathrm{~Gy}$. All patients received concomitant chemotherapy and two neoadjuvant chemotherapy. The median follow-up for all patients was 40months; one patient developed an immobility of the soft palate and another had grade II trismus.

Conclusion: Brachytherapy alone or after EBRT may play an important role in reirradiation of locally recurrent NPC with acceptable toxicity.

Keywords: nasopharyngeal carcinoma, brachytherapy, local recurrence, reirradiation
Volume 3 Issue $6-2017$

Toulba A, Bakkali H, Diabate H, Kebdani H, EL-Gueddari BK, Benjaafar N

Department of Radiotherapy, National Institute of Oncology, Morocco

Correspondence: Toulba A, Department of Radiotherapy, National Institute of Oncology, Morocco,

Email ahmedoux@gmail.com

Received: September 23, 2016 | Published: August 08, 2017
Abbreviations: UCNT, undifferentiated carcinoma of nasopharyngeal type; EBRT, external beam radiotherapy; HDR, high dose rate; CT, computed tomography

\section{Case report}

Nasopharyngeal cancer is a most cancer of head and neck oncology in North Africa and South East Asia. The predominant histological type is represented by an undifferentiated carcinoma of nasopharyngeal type (UCNT). The risk of local recurrence after treatment and local distance is higher 13 to $30 \%$ at 2 years. ${ }^{1,2}$ Patients, who develop local recurrence, salvage treatment remains a very difficult challenge due to the proximity to critical structures. Management of recurrences includes external radiotherapy or external radiation with a boost delivered by brachytherapy, chemotherapy, surgery, radiosurgery, or combinations of these methods. ${ }^{3-11}$ No randomized trials have been performed to evaluate the therapeutic ratio of different treatment methods. Brachytherapy provides advantages to treat the small tumor with very high doses and protects surrounding healthy tissues. ${ }^{12}$ Through these cases we studied the contribution of brachytherapy in reirradiation for local recurrence of NPC (Figure 1).

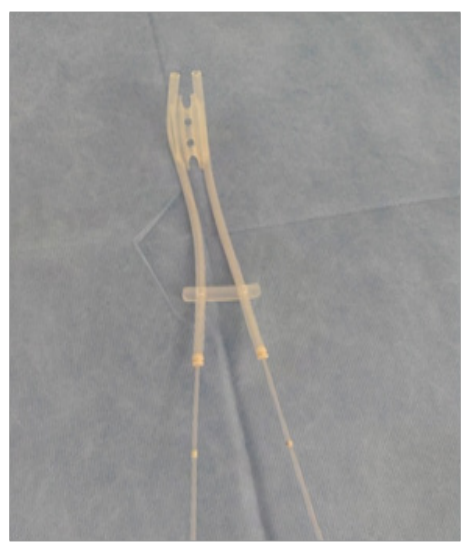

Figure I Rotterdam nasopharyngeal application produced by elekta/ nucletron.

\section{Patients and methods}

From 2002 to 2011, five patients were reirradiated for local recurrence nasopharynx cancer. All patients were irradiated by 
exclusive brachytherapy or association EBRT and brachytherapy HDR.

\section{Patients and technique}

Patient characteristics: The median age was 43years, all were male, four patients had World Health Organization Class III histology, and one with nasopharyx cancer type I. Two, 1 and 2 patients had recurrent Stage I, II, III, respectively. Median time to reirradiation was 3years Table 1. Recurrence was suspected clinically, imaging by magnetic resonance nasopharyngeal was performed in two patients and a nasopharyngeal CT scan in two patients. Confirmation was made by biopsy for all cases. Four patients received combined-modality treatment, consisting of external beam radiotherapy (EBRT) with chemotherapy followed by intracavitary brachytherapy High Dose Rate (HDR) boost, one patient received intracavitary brachytherapy alone for dosimetric constraints for organs at risk. The total radiation dose EQD2 was between 60 and 70Gy. All patients received concomitant chemotherapy and two neoadjuvant chemotherapy Table 2.

Table I Patient characteristics

\begin{tabular}{llllll}
\hline Patient & I & $\mathbf{2}$ & $\mathbf{3}$ & $\mathbf{4}$ & $\mathbf{5}$ \\
\hline Age & 35 & 50 & 56 & 69 & 38 \\
Sex & M & M & M & M & M \\
Histology & WHO & WHO & WHO & WHO & WHO \\
$\begin{array}{llll}\text { Stage (TNM 2002) } \\
\text { III }\end{array}$ & IIB & III & III & I & III \\
$\begin{array}{l}\text { Interval to } \\
\text { reirradiation } \\
\text { (months) }\end{array}$ & 45 & 98 & 38 & 34 & 30 \\
\hline
\end{tabular}

Table 2 Treatment

\begin{tabular}{lccccc}
\hline Patient & I & $\mathbf{2}$ & $\mathbf{3}$ & $\mathbf{4}$ & $\mathbf{5}$ \\
\hline EBRT & $\mathrm{Y}$ & $\mathrm{N}$ & $\mathrm{Y}$ & $\mathrm{Y}$ & $\mathrm{Y}$ \\
HDR & $\mathrm{Y}$ & $\mathrm{Y}$ & $\mathrm{Y}$ & $\mathrm{Y}$ & $\mathrm{Y}$ \\
Concomitant chemotherapy & $\mathrm{Y}$ & $\mathrm{Y}$ & $\mathrm{Y}$ & $\mathrm{Y}$ & $\mathrm{Y}$ \\
Neoadjuvant chemotherapy & $\mathrm{Y}$ & $\mathrm{N}$ & $\mathrm{Y}$ & $\mathrm{N}$ & $\mathrm{N}$
\end{tabular}

$\mathrm{N}$, no; Y, yes

\section{Technique \\ EBRT}

Patients treated using 3D-CRT (Three Dimensional Conformal Radiotherapy); volume was usually confined to the site of recurrence in the nasopharynx with a $1-2 \mathrm{~cm}$ margin. Treatment was delivered at $1.8-2 \mathrm{~Gy} /$ fraction daily, 5 days a week (Table 3 ).

\section{Brachytherapy}

The applicator used was the Rotterdam nasopharyngeal made out of soft silicone, suitable for application with stepping source afterloaders for HDR brachytherapy. The application is easy, after local anesthesia with $2 \%$ xylocaine liquid. A flexible guide wire was introduced into one nasal cavity; the end was recuperated with a forceps into the oropharynx and brought outside the mouth. The procedure was repeated at the other side. The applicator was then advanced over the guide wires, fixed to them by clamps and pulled gently through the mouth and the oropharynx into the nasopharynx. The legs of the applicator sort through the nostrils and fixed with a silicone bridge, pushed against the nasal septum. The applicator tubes are then brought into the applicator and fixed.

Localization films were taken to determine the position of the applicator; radiological images were made in the anterior-posterior and lateral planes. After a plan had been completed and accepted, catheters were introduced into the applicator and then connected by of transferring cables to the Microselectron HDR produced by Elekta/Nucletron (Ir 192). The patient remained in the room of treatment during a few minutes. The applicator was removed from the nasopharynx following the completion of the whole course of treatment. Whenever necessary, analgesic substances, steroids and irrigation were used in order to improve treatment tolerance.

\section{Results}

\section{Retreatment}

Local control: The total radiation dose EQD2 was between 60 and 70. The median follow-up after reirradiation was 42 months. Local control was observed in all cases. All patients are still alive.

Late complications: All patients had xerostomia, one patient had an immobility of the soft palate, another had grade II trismus and one had bilateral temporal lobe necrosis after the first irradiation.

\section{Discussion}

Our study suggests that brachytherapy can be effective for local recurrence reirradiation in spite of the toxicity due to the proximity of already irradiated critical structures. Age, histology, recurrence T-classification, and interval to recurrence were factors for overall survival. ${ }^{1,8,13}$ Several publications reported the lymph node spread and reirradiation dose are one of the most important factors for localregional progression after the salvage treatment;, ${ }^{9,14,15}$ the general consensus is that reirradiation dose above 60 Gray (Gy) is needed for effective salvage. ${ }^{2}$ Brachytherapy is an option for treating located small tumors. In the literature, EBRT remains a main treatment method to be used. Many authors combined external RT with a localized boost (using brachytherapy or SRS/FSRT) to achieve high tumor dose with better sparing of adjacent organs at risk. ${ }^{8,9}$ Qiu et al. ${ }^{8}$ in a retrospective study reported the result of different reirradiation techniques for local recurrence NPC, 168 patients were treated with four different irradiation modalities (3D-CRT, IMRT, BT, and BT with external radiotherapy), and $43 \%$ have received brachytherapy. The year LRRFS, DFS and OS were 82.58\%, 53.41 and 37.99\%, respectively. In agreement with our study, this study reported that all patients had xerostomia. Fatal complication because of excessive nasal bleeding was reported in ten cases.

The Hong Kong Nasopharyngeal Carcinoma Study Group ${ }^{16}$ reported in retrospective study the rate of survival rate patients with isolated local failure was $74 \%, 4$ patients died in consequence of radiotherapy complication. The late toxicity is a limiting factor for reirradiation. Pryzant et al. ${ }^{17}$ showed that the incidence of severe late toxicity increased significantly when the total dose by the 2 courses of RT exceeded 100 Gy versus those with $<100$ Gy ( $39 \%$ vs. $4 \%$ at 5 year). Lee et al. ${ }^{18}$ showed that late complications were were predominantly affected by the initial dose, the interval between the two courses and the fractionation schedules. Development of techniques with increasing conformity brings promising opportunities for attaining 
high tumor dose with better protection of normal tissues. Lengyel and al found that necrosis of the soft palate were observed in $10 \%$ of patients; this is consistent with our results. Kwong et al. ${ }^{19}$ seen after brachytherapy by gold grain implantation symptoms that included headache, palatal fistula, and mucosal radiation necrosis at the site of implantation in $28.3 \%, 18.9 \%$, and $16 \%$, respectively. Late toxicity, especially neurological damage is a serious concern. In conclusion, Brachytherapy alone or after EBRT may play an important role in reirradiation of locally recurrent nasopharynx cancer to decrease the dose to the organs at risk already irradiated. ${ }^{20-25}$

\section{Acknowledgements}

None.

\section{Conflict of interest}

Author declares that there is no conflict of interest.

\section{References}

1. Suárez C, Rodrigo J, Rinaldo A, et al. Current treatment options for recurrent nasopharyngeal cancer. Eur Arch Otorhinolaryngol. 2010;267(12):1811-1824.

2. Lee A, Fee W, Ng W, et al. Nasopharyngeal carcinoma: salvage of local recurrence. Oral Oncol. 2012;48(9):768-774.

3. Wei W, Chan J, Ng R, et al. Surgical salvage of persistent or recurrent nasopharyngeal carcinoma with maxillary swing approach - Critical appraisal after 2 decades. Head Neck. 2011;33(7):969-975.

4. Chua D, Wei W, Sham J, et al. Treatment outcome for synchronous locoregional failures of nasopharyngeal carcinoma. Head Neck. 2003;25(7):585-594.

5. Chua D, Sham J, Au G. Induction chemotherapy with cisplatin and gemcitabine followed by reirradiation for locally recurrent nasopharyngeal carcinoma. Am J Clin Oncol. 2005;28(5):464-471.

6. Zheng X, Chen L, Chen Y, et al. Three-dimensional conformal radiotherapy versus intracavitary brachytherapy for salvage treatment of locally persistent nasopharyngeal carcinoma. Int J Radiat Oncol Biol Phys. 2004;60(1):165-170.

7. Chua D, Wu S, Lee V, et al. Comparison of single versus fractionated dose of stereotactic radiotherapy for salvaging local failures of nasopharyngeal carcinoma: a matched-cohort analysis. Head Neck Oncol. 2009;1:13.

8. Qiu S, Lu J, Zheng W, et al. Advantages of intensity modulated radiotherapy in recurrent T1-2 nasopharyngeal carcinoma: a retrospective study. BMC Cancer. 2014;14:797.

9. Cheah S, Lau F, Yusof M, et al. Treatment outcome with brachytherapy for recurrent nasopharyngeal carcinoma. Asian Pac J Cancer Prev 14(11):6513-6518.
10. Ozyigit G, Cengiz M, Yazici G, et al. A retrospective comparison of robotic stereotactic body radiotherapy and three-dimensional conformal radiotherapy for the reirradiation of locally recurrent nasopharyngeal carcinoma. Int J Radiat Oncol Biol Phys. 2011;81(4):e263-e268.

11. Wei W, Kwong D. Recurrent nasopharyngeal carcinoma: surgical salvage vs. additional chemoradiation. Curr Opin Otolaryngol Head Neck Surg. 2011;19(2):82-86.

12. Koutcher L, Lee N, Zelefsky M, et al. Reirradiation of locally recurrent nasopharynx cancer with external beam radiotherapy with or without brachytherapy. Int J Radiat Oncol Biol Phys. 2010;76(1):130-137.

13. Hwang J, Fu K, Phillips T. Results and prognostic factors in the retreatment of locally recurrent nasopharyngeal carcinoma. Int J Radiat Oncol Biol Phys. 1998;41(5):1099-1111.

14. Chan Y, Lee V, Chow V, et al. Extracapsular lymph node spread in recurrent nasopharyngeal carcinoma. Laryngoscope. 2011;121(12):2576-2580.

15. Smee R, Meagher N, Broadley K, et al. Recurrent nasopharyngeal carcinoma: current management approaches. Am J Clin Oncol. 2010;33(5):469-473.

16. Yu K, Leung S, Tung S, et al. Survival outcome of patients with nasopharyngeal carcinoma with first local failure: a study by the Hong Kong Nasopharyngeal Carcinoma Study Group. Head Neck. 2005;27(5):397-405.

17. Pryzant R, Wendt C, Delclos L, et al. Re-treatment of nasopharyngeal carcinoma in 53 patients. Int J Radiat Oncol Biol Phys. 1992;22(5):941-947.

18. Lee N, Hoffman R, Phillips T, et al. Managing nasopharyngeal carcinoma with intracavitary brachytherapy: one institution's 45-year experience. Brachytherapy. 2002;1(2):74-82.

19. Kwong D, Wei W, Cheng A, et al. Long term results of radioactive gold grain implantation for the treatment of persistent and recurrent nasopharyngeal carcinoma. Cancer. 2001;91(6):1105-1113.

20. Maalej M, Ben A, Kochbati L, et al. Brachytherapy for primary and recurrent nasopharyngeal carcinoma: treatment techniques and results. Cancer Radiother. 2007;11(3):117-121.

21. Lengyel E, Baricza K, Somogyi A, et al. Reirradiation of locally recurrent nasopharyngeal carcinoma. Strahlenther Onkol. 2003;179(5):298-305.

22. Hall C, Harris R, A’Hern R, et al. Le Fort I osteotomy and low-dose rate Ir192 brachytherapy for treatment of recurrent nasopharyngeal tumours. Radiother Oncol. 2003;66(1):41-48.

23. Fischer M, Stüben G, Stuschke M, et al. Brachytherapy with (192) Iridium in the treatment of recurrent nasopharyngeal carcinoma. Laryngorhinootologie. 2002;81(2):106-110.

24. Leung T, Tung S, Sze W, et al. Salvage radiation therapy for locally recurrent nasopharyngeal carcinoma. Int J Radiat Oncol Biol Phys. 2000;48(5):1331-1338.

25. Syed A, Puthawala A, Damore S, et al. Brachytherapy for primary and recurrent nasopharyngeal carcinoma: 20years' experience at Long Beach Memorial. Int J Radiat Oncol Biol Phys. 2000;47(5):1311-1321. 